\title{
An Extremely Effective and Safe Approach of Guiding Catheter Crossing over Spasmodic Radial or (and) Brachial Artery in Patients Whose Percutaneous Coronary Intervention Was Undergone via Radial Artery Access
}

\author{
Zhuhua $\mathrm{Ni}^{*}$, Lefeng Wang1, Xinchun Yang1, Junping Deng2 ${ }^{2}$ Jianhong Zhao ${ }^{3}$, Jiqiang Zhang4, \\ Shuying Qi ${ }^{5}$, Tao Zhang6, Yong $\mathrm{Li}^{7}$ \\ ${ }^{1}$ Heart Center, Beijing Chaoyang Hospital, Institute of Cardiovascular Disease, Capital Medical University, Beijing, China \\ ${ }^{2}$ Linfen People's Hospital, Shanxi, China \\ ${ }^{3}$ Jincheng Heart and Brain Disease Hospital, Shanxi, China \\ ${ }^{4}$ Linfen Second People's Hospital, Shanxi, China \\ ${ }^{5}$ Yantai Haigang Hospital, Shangdong, China \\ ${ }^{6}$ Ningjun People's Hospital, Shandong, China \\ ${ }^{7}$ Leling People's Hospital, Shandong, China \\ Email: *nizhuhua@sina.com
}

How to cite this paper: Ni, Z.H., Wang, L.F., Yang, X.C., Deng, J.P., Zhao, J.H., Zhang, J.Q., Qi, S.Y., Zhang, T. and Li, Y. (2018) An Extremely Effective and Safe Approach of Guiding Catheter Crossing over Spasmodic Radial or (and) Brachial Artery in Patients Whose Percutaneous Coronary Intervention Was Undergone via Radial Artery Access. World Journal of Cardiovascular Diseases, 8, 169-182. https://doi.org/10.4236/wjcd.2018.82017

Received: January 29, 2018

Accepted: February 21, 2018

Published: February 24, 2018

Copyright $\odot 2018$ by authors and Scientific Research Publishing Inc. This work is licensed under the Creative Commons Attribution International License (CC BY 4.0).

http://creativecommons.org/licenses/by/4.0/ (c) (i) Open Access

\begin{abstract}
Objective: Percutaneous coronary intervention (PCI) via the radial artery access has more advantages than that of femoral artery access, while radial or (and) brachial artery have tendency to be spasmodic. We sought to investigate the effectiveness and safety of guiding catheter crossing over spasmodic radial or (and) brachial artery segments by the aid of PCI wire and balloon compared with traditional anti-spasmodic approach. Methods: The clinical data of 168 patients with coronary artery disease (CAD) (group A), whose PCI was performed via radial artery access with radial or (and) brachial artery spasm or (and) dissection and guiding catheter passing through spasmodic segments successfully by the aid of PCI guiding wire and balloon were analyzed retrospectively, simultaneously, the other 73 patients (group B) who used conventional approach to cross over the spasmodic radial or (and) brachial artery segments were treated as the control. The success rate, the time consumption and the complication were compared between the two groups. Findings: There was no significant difference in the spasmodic site between the two groups (all $\mathrm{p}$ value $>0.05$ ). The success rate in group A was significantly
\end{abstract}


higher than that in group B $(168(100 \%)$ vs $28(38.4 \%), \mathrm{p}<0.0001)$. As for those of successful crossover in the two groups, the time consumption of guiding catheter crossing over spasmodic segment in group A was shorter than that in group $B(p<0.0001)$. The incidence of forearm hematoma in group A was lower than that in group B $(7(4.2 \%)$ vs $14(19.2 \%), \mathrm{p}<0.0001)$. Conclusions: It is more effective and safer for guiding catheter crossing over spasmodic or (and) dissected radial or (and) brachial artery segments by the aid of PCI guiding wire and balloon than using the routine approach of administration of anti-spasm drugs for trans-radial PCI.

\section{Keywords}

Trans-Radial PCI, Radial or (and) Brachial Artery Spasm, Guiding Catheter Crossover of Spasmodic Segment, Guiding Wire and Balloon Assistance

\section{Introduction}

There are many advantages when percutaneous coronary intervention (PCI) is undergone via radial artery (RA) access compared to femoral artery access for patients with coronary artery disease (CAD). Less vessel puncture-related complications, less pain and more willingness of the patients and shorter hospitalization time for PCI via radial artery access make it the most often adopted vessel access in current clinical practice for PCI [1]. Comparing with femoral artery, radial artery or (and) brachial artery (BA) have tendency to be spasmodic. The success rate of the traditional anti-spasmodic method, i.e., anti-spasmodic drugs, such as nitroglycerine, nitroprusside, diltiazem, etc., to relieve radial or (and) brachial artery spasm is relatively low. Once spasm cannot be relieved, the guiding catheter is hard to cross over the spasmodic artery, and even severe dissection or hematoma occurs in the upper limb [1]. Under the circumstances, femoral artery, contralateral radial or ulnar artery has to be punctured. It will certainly aggravate the pain, prolong procedural and hospitalization time, and impose economic burden of the patients (especially femoral artery is punctured and vessel suture instrument is used) [2]. To date, it has been demonstrated in our experience that the problem can be solved by the aid of PCI guiding wire and balloon effectively and safely. It is our strong recommendation to apply this approach to cross over spasmodic radial artery or (and) brachial artery when PCI is undergone via radial artery access.

\section{Subjects and Methods}

\subsection{Study Subjects}

The clinical data of a total of 241 patients with coronary heart disease whose PCI was performed via the radial artery access with radial or (and) brachial artery spasm from January 2014 to January 2017 in seven hospitals across China (168 
cases in group A were applied PCI wire and balloon-guided spasmodic segment crossover, and 73 cases in group B were applied routine methods of administration of anti-spasm drugs to cross over the spasmodic segment) were analysed and compared retrospectively so as to observe the success rate and complications between the two groups and to identify the advantages and disadvantages of the two methods. The inclusion criteria: 1) patients whose coronary angiography was performed via the radial artery access and the coronary lesions were suitable for PCI; 2) PCI guiding catheter could not cross radial or brachial artery and radial artery angiography confirmed that radial or (and) brachial artery spasm occurred. Exclusion criteria: 1) severe brandy cardia (heart rate $<50 / \mathrm{min}$ ); 2) hypotension (blood pressure $<90 / 60 \mathrm{mmHg}$ ) or cardiogenic shock; 3 ) anatomical abnormality of radial or brachial artery, e.g. radial or brachial artery loop existed.

Generally, a 6F guiding catheter was chosen for trans-radial artery PCI; however, as for some complex cases, such as bifurcation lesions, a 7F guiding catheters can also be selected according to the situation, while a 7F guiding catheter was not routinely applied in patients of thin body size.

\subsection{Identification of Radial or (and) Brachial Artery Spasm via the Radial Arterial Access}

1) Operators felt resistance when manipulating interventional instruments (such as the guiding wire, angiographical catheter, or guiding catheter), or cannot push forward the interventional instruments; 2) Patients may feel swelling pain in the forearm; 3) The injection of contrast medium through the arterial sheath or the catheter revealed focal or diffuse contraction of radial artery or (and) brachial artery, i.e. the vessel became narrower in diameter and contrast medium exosmosis was often seen.

\subsection{Key Operation Points of PCI Wire and Balloon-Guided Spasmodic Segment Crossover}

1) All radial and (or) brachial artery spasms should be confirmed by injection of contrast medium through the arterial sheath or the catheter. Once the spasm was found , $100-300 \mu \mathrm{g}$ of nitroglycerine was injected through the sheath or guiding catheter according to the blood pressure at the operator's discretion (not necessary); then a guiding catheter was adjusted and positioned by the aid of one hydrophilic wire (so-called the "Loach" wire, 0.035" in diameter, 1 inch $=2.54$ $\mathrm{cm}$ ) so as the tip of guiding catheter was away from the beginning of the spasmodic segment for at least $10 \mathrm{~mm}$. 2) One soft-tip PCI guiding wire (0.014" in diameter), such as BMW(Abbott, USA) or Runthrough (Terumo, Japan) was manipulated carefully to cross over the spasmodic segment. 3) According to the size of guiding catheter, an appropriate balloon was then selected. Generally, a $6 \mathrm{~F}$ guiding catheter was selected for PCI via trans-radial artery access in most cases, so a predilation balloon of $2.0 \mathrm{~mm}$ in diameter (e.g. Sprinter, Medtronic, 
USA; Sequent, Braun, USA, etc ) should be selected for the matching. Sometimes a 7F guiding catheter may be selected for complex PCI, then a balloon of $2.5 \mathrm{~mm}$ in diameter should be selected for the matching. The balloon length should not be too short, and the best length is about $15 \mathrm{~mm}$ or $20 \mathrm{~mm}$. 4) Under the X-ray guidance, the balloon selected was deliver to the catheter tip through the guiding wire ( 0.014 " in diameter), during which the key point was to push only $1 / 2$ of the balloon outside the guiding catheter while the rest half was kept inside the catheter. 5) After the balloon was expanded with $6-8$ atm $(1 \mathrm{~atm}=101.325 \mathrm{kPa})$, the operator must push the guiding catheter (rather than the outer rod of the balloon catheter, we called it as balloon- guiding catheter complex (BGCC)) slowly, then the BGCC will go forward simultaneously to pass through the spasmodic segment eventually, at which time no obvious or slight resistance could be felt; after passing through the spasmodic segment, the resistance will be further reduced. If the resistance during the pushing process was large, no force should be applied for the crossover. Once it was confirmed that the catheter had crossed over the spasmodic segment according to the hand feeling, the operator should stop pushing the guiding catheter, and then deflate the balloon. When confirmed the complete deflation by X-ray, the balloon should be retracted to the guiding catheter, followed by re-angiography via the guiding catheter. If the catheter confirmatively passed through the spasmodic segment, the balloon should be withdrawn; the PCI wire can be withdrawn at the same time or still remained in the catheter. 6) The "Loach" slippery guiding wire (preferred) or the "Green" guiding wire was then used to complete the placement of the guiding catheter. 7) After completing PCI, the "Loach" guiding wire was then re-inserted so as to assist the careful withdrawal of the guiding catheter back to the distal end of the spasmodic segment and re-angiography was needed. If angiography revealed no contrast medium extravasation in the spasmodic segment, the guiding catheter and sheath can be directly removed; if contrast medium extravasation was found, elastic bandage should be applied at the site of contrast medium extravasation (Figures 1-3).

\subsection{Routine Crossover Method}

1) If vasospasm was confirmed by angiography, the guiding catheter cannot be pushed forward, then one 0.035 " guiding wire (the "Loach") should be used to assist the withdrawal of the guiding catheter outside the body. 2) According to the blood pressure, 100 - $300 \mu \mathrm{g}$ of nitroglycerine was injected through the sheath, and after one minute to several minutes, the contrast medium should be re-injected through the sheath to observe whether spasm was lifted: If not, the above procedure should be repeated. If the effect of nitroglycerine was poor, 100 $300 \mu \mathrm{g}$ of verapamil (each time) or $100-300 \mu \mathrm{g}$ of diltiazem (each time) can be administrated via the sheath according to blood pressure and heart rate, followed by confirming the relief of spasm by contrast medium injection via the sheath. If necessary, the above drugs can be administrated via the systemic veins. 


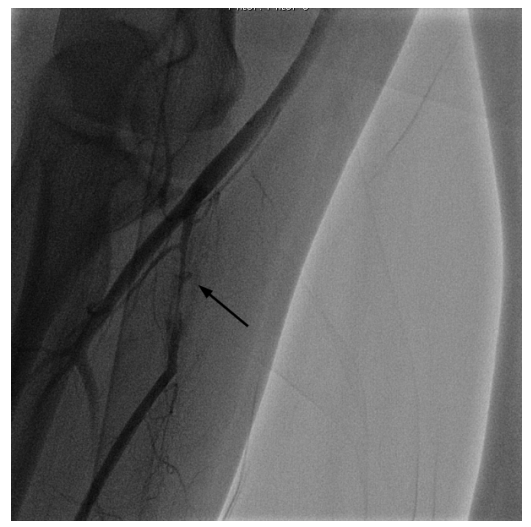

(a)

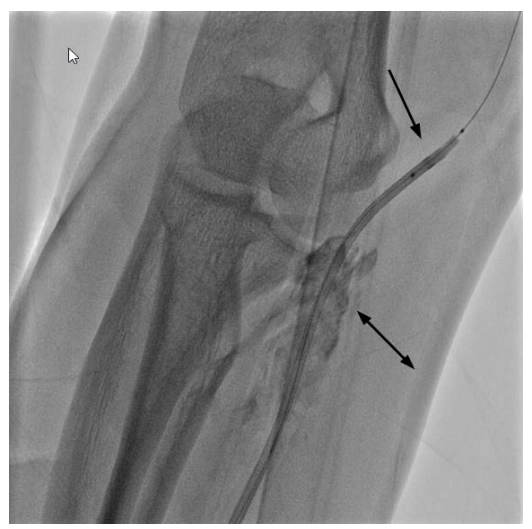

(c)

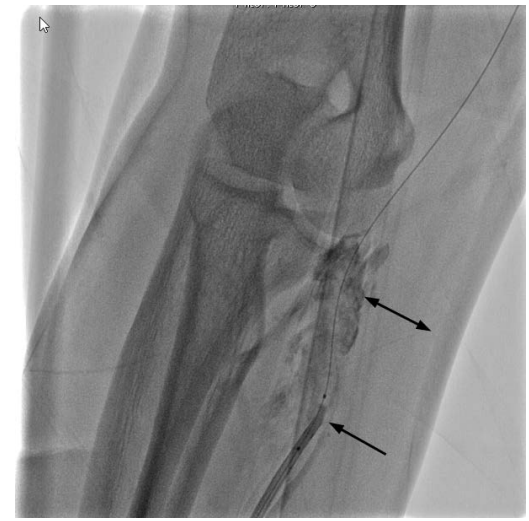

(b)

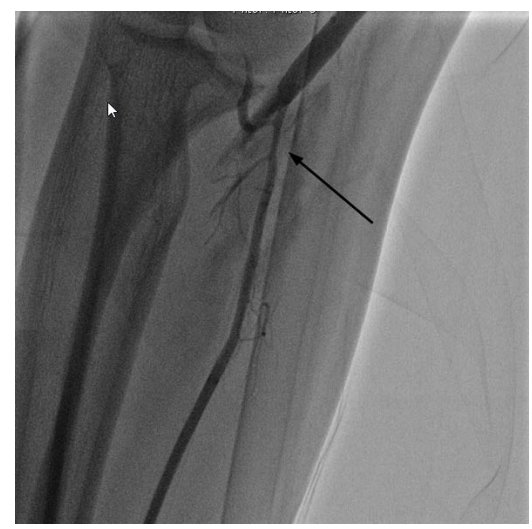

(d)

Figure 1. Male, 43 years old, radial artery spasm together with severe dissection. (a): Radial artery spasm together with severe dissection (arrow). (b): A BMW guiding wire successfully passed through the spasmodic dissection segment, the half of the balloon (2.0 $\mathrm{mm} \times 15 \mathrm{~mm}$ ) was positioned in the guiding catheter and the rest was kept in the blood vessel and was dilated with $8 \mathrm{~atm}$ (single arrow); significant exudation of the contrast medium could be observed locally (Double arrow). (c): When maintaining the filling status of the balloon, the guiding catheter was pushed in vitro (rather than the outer rod of the balloon), then the guiding catheter and balloon, as a whole (namely the balloonguiding catheter complex, BGCA), successfully passed through the dissected segment (single arrow); the exudation of the contrast medium can be observed locally (double arrow). (d): After PCI, the original spasmodic segment was significantly relieved, the dissected segment was closed, and no contrast medium exudate could be observed (arrow).

3) If spasm was found relieved or reduced by angiography, the "Loach" wire should be used to assist the crossover of guiding catheter. If any obvious resistance was encountered, the contrast medium should be injected instead of forcing the catheter to pass through, and if no contrast medium exosmosis was found, the above procedure can be continued until the catheter passed through the spasmodic segment. If the extravasation of the contrast medium was found, elastic bandage should be applied immediately, and the contralateral radial artery or femoral artery should be selected for the puncture.

\subsection{Statistical Analysis}

Data were analyzed using the SPSS Statistical Package, version 21.0. Categorical 


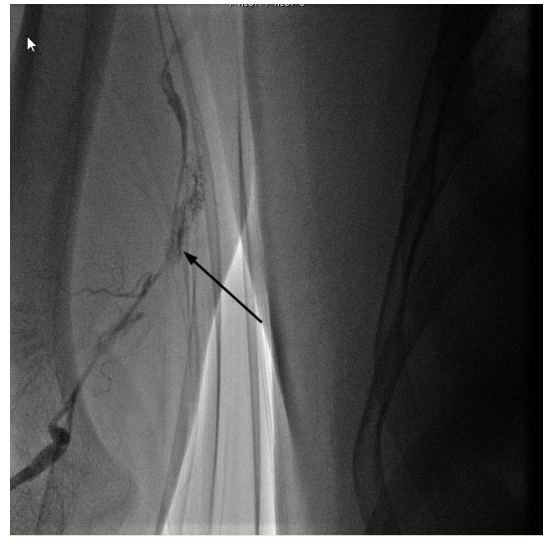

(a)

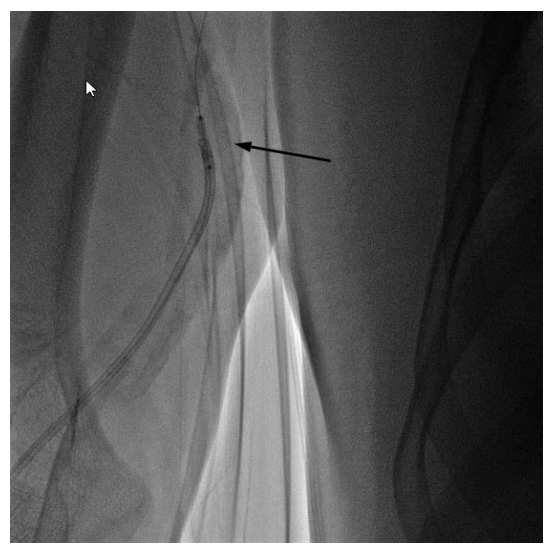

(c)

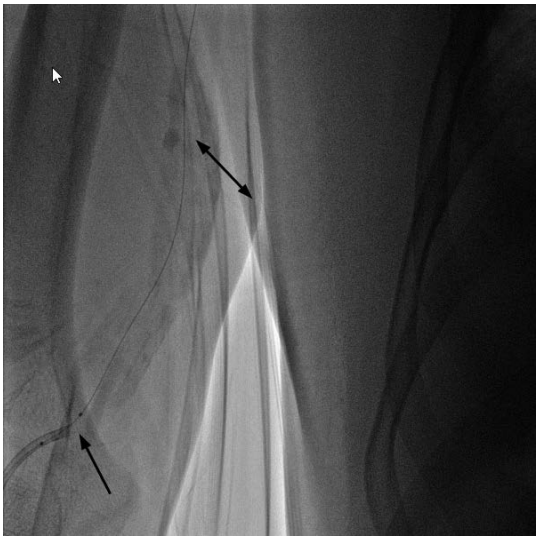

(b)

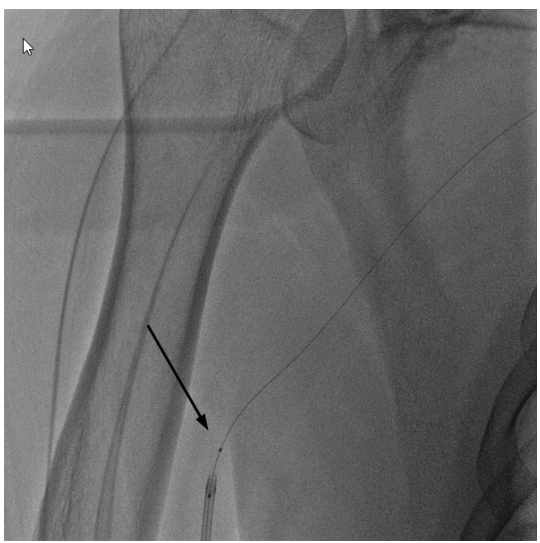

(d)

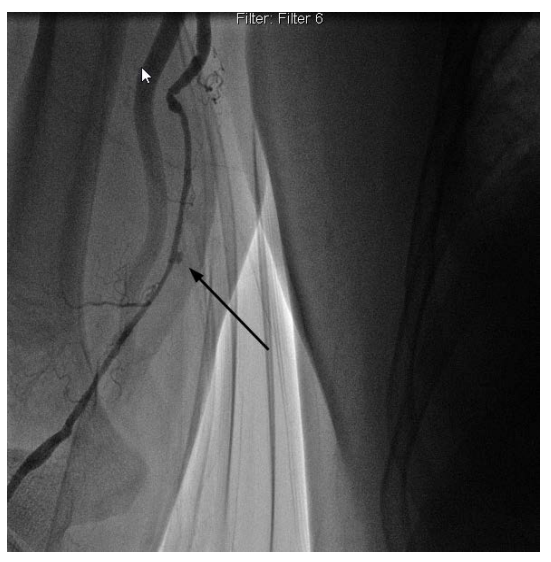

(e)

Figure 2. Female, 63 years old, brachial artery spasm together with severe dissection. (a): Resistance was encountered when pushing the guiding catheter, angiography revealed brachial arterial tortuosity, severe spasm combined with dissection, nearly occlusion. (b): A BMW guiding wire successfully crossed over the spasmodic and dissected segment, the half of the balloon was positioned in the guiding catheter and the rest was kept in the blood vessel, together with 8-atm expansion (single arrow). (c): BGCA passed through the dissected segment smoothly (single arrow). (d): After passing through the dissected segment, the balloon was deflated and guiding wire was withdrawn (single arrow). (e): After PCI, the original spasmodic segment was significantly relieved, and the dissection almost completely closed; no more contrast medium exudate can be observed (single arrow). 


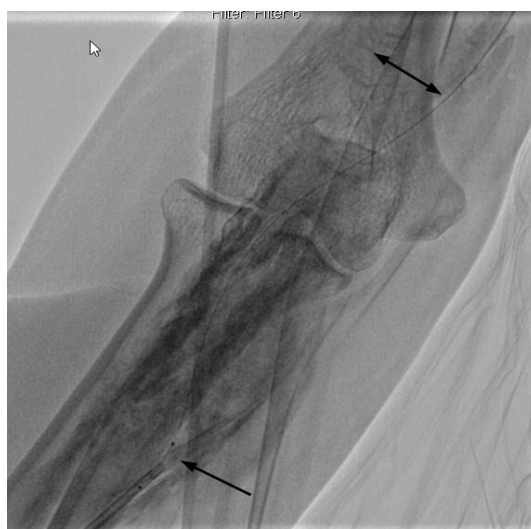

(a)

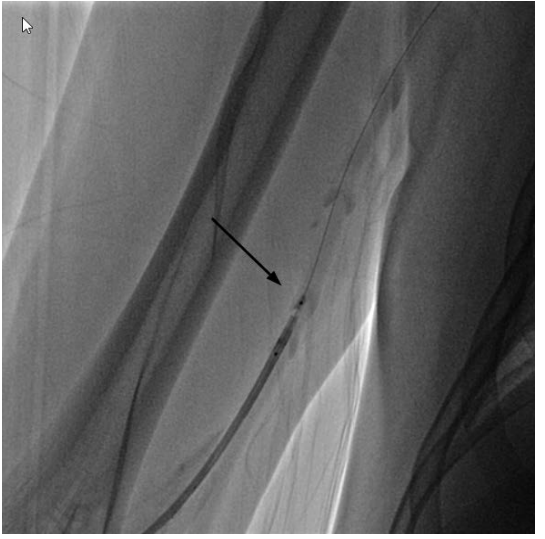

(c)

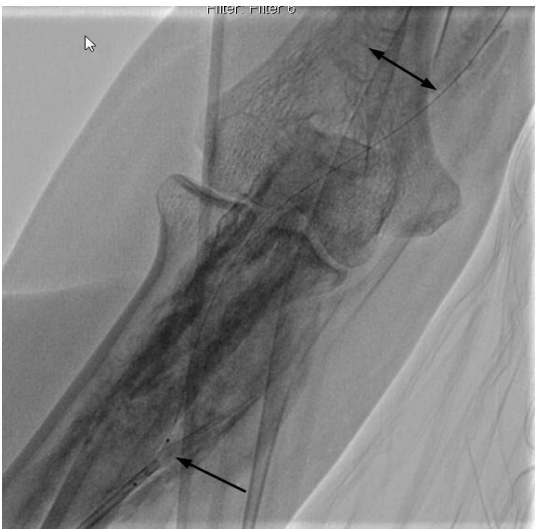

(b)



(d)

Figure 3. Male, 66 years old, spasm in the radial artery and brachial artery together with severe dissection. (a): Spasm in the radial artery and brachial artery together with severe dissection, almost occlusive (between the single arrow and double arrow). (b): The BMW wire was damaged when attempting to pass through the spasmodic segment, while the Runthrough guiding wire successfully passed through (double arrow); the half of the balloon was placed in the guiding catheter and the rest was kept in the blood vessel, and expanded using $8 \mathrm{~atm}$ (single arrow). (c): BGCA passed through the dissected segment successfully (single arrow). (d): After PCI, spasm in the brachial artery and radial artery almost disappeared, and the dissection was closed, no contrast medium exudation can be observed; local vascular stenosis and tortuosity can be observed (double arrow); the BGCA could pass through the tortuous and stenosis segment even if relatively large resistance was encountered.

variables were expressed as frequencies (n (\%)) analyzed using Chi-square test; continuous variables were presented as means \pm Standard Deviations (SDs) and analyzed using the two-tailed Student's t-test. A $p$ value of $<0.05$ was considered statistically significant.

\section{Results}

\subsection{Baseline Characteristics between the Two Groups}

There were no statistical differences in age, sex, hypertension, diabetes mellitus, current smoker, unstable angina pectoris, myocardial infarction between the two groups (all p > 0.05) (Table 1). 
Table 1. Baseline clinical characteristics.

\begin{tabular}{cccc}
\hline Characteristic & Group A & Group B & p value \\
\hline Male sex, n (\%) & N = 168 & N = 73 & 0.809 \\
Mean age (years \pm SD) & $88(52.4)$ & $37(50.7)$ & 0.741 \\
Hypertension, n (\%) & $124(73.8)$ & $54(74.0)$ & 0.979 \\
Diabetes mellitus, n (\%) & $99(58.9)$ & $42(57.5)$ & 0.840 \\
Alcohol, n (\%) & $38(22.6)$ & $20(27.4)$ & 0.762 \\
Current smoker, n (\%) & $76(45.2)$ & $34(46.5)$ & 0.848 \\
Unstable angina pectoris, n (\%) & $146(86.9)$ & $67(91.8)$ & 0.278 \\
Acute myocardial infarction, n (\%) & $\mathbf{2 2 ( 1 3 . 1 )}$ & $\mathbf{6 ( 8 . 2 )}$ & $\mathbf{0 . 2 7 7}$ \\
\hline
\end{tabular}

\subsection{Comparison of Spasmodic Sites, Complication, Success Rate and Time Consumption for Guiding Catheter Crossover between the Two Groups}

There was no significant difference in the spasmodic segment between the two groups (all $\mathrm{P}>0.05$ ), but the incidence of brachial artery spasm was higher than that of radial artery in the two groups. 1) All the PCI guiding wires used in group A successfully passed through the spasmodic or (and) the dissected segment of blood vessel, and all guiding catheters passed through successfully. Except for five cases in which two guiding wires were applied (if a BMW wire failed to cross, then a Runthrough wire was applied, vice versa), the rest 163 cases were applied only one guiding wire, among which the BMW guiding wire was used in 102 cases and the Runthrough guiding wire was used in 61 cases. Significant resistance was encountered in one of the five cases using two guiding wires when trying to pass through the dissected site with a BMW guiding wire. It was almost trapped in the dissection sites when pushing forward and withdrawing the guiding wire. The tip of wire was found tightly knotted when removed from the body, so one Runthrough guiding wire was chosen and then passed through the spasmodic and dissected segment relatively smoothly; The reason why the other four cases using two wires also was that the wire tip was found damaged and deformed and failed to pass through the spasmodic and dissected segment, then changed for another different wire and passed through eventually. 2) Only 28 cases in group B exhibited successful crossover of the spasmodic blood vessel which took a long time. That the patient with the longest time to cross over the spasmodic site was due to weak pulse in the contralateral radial artery and the patient's resolute refusal of puncturing the femoral artery. It took nearly $40 \mathrm{mi}-$ nutes for the catheter crossing over the spasmodic segment.

Among the 45 unsuccessful cases in group B, 34 cases exhibited varying degrees of contrast medium exudation, which suggested vascular dissection and then other paths were selected immediately. The contrast medium exudation site was instantly applied elastic bandage. There were 121 (121/168) cases exhibiting varying degrees of contrast medium exudation in group $A$, which suggested 
vascular dissection. For those patients, no elastic bandage was immediately needed if the guiding catheter could cross over the dissected segments within several minutes. If there was serious exudation and guiding catheter could not cross over the dissected segments rapidly, then the elastic bandage should be applied. Four patients were the case in group A. After PCI was completed, angiography was needed for all cases in order to observe if contrast medium exudation still existed in the previously dissected segments. It was demonstrated that contrast medium exudation disappeared completely or nearly disappeared and no contrast medium exuded any more in all cases in group A. In group B, 14 (19.2\%) patients exhibited postoperative hematoma, so the complication was tremendously lesser in group A than group B (4.2\% vs $19.2 \%, \mathrm{p}<0.0001)$. The hospital stay was shorter in group A than group B $(7.2 \pm 5.7$ vs $8.8 \pm 4.9, \mathrm{p}=$ 0.016). Generally, for patients with postoperative hematoma, the hospital stay would prolong for 2 to 4 days.

The time consumption of guiding catheter crossing over spasmodic segment was much shorter in patients of group A than that of group B $(p<0.0001)$. The majority of cases were less than five minutes in group $\mathrm{A}$, on the contrary, most of cases were more than five minutes in group B (Table 2).

A 7F guiding catheter was chosen in five patients in group $A$ and two patients in group B, respectively, and one $2.5 \mathrm{~mm}$-in-diameter balloon was then selected so as to better match the diameter of the guiding catheter. In some cases, the angiography could be completed by a $5 \mathrm{~F}$ catheter successfully without being aware of the existence of radial or (and) brachial artery spasm. If PCI needed, a 6F guiding catheter and a $2.5 \mathrm{~mm}$-in-diameter predilation balloon may be chosen.

Table 2. Spasmodic sites, complication, success rate and time consumption for guiding catheter crossover the spasmodic segment of the two approaches.

\begin{tabular}{cccc}
\hline Parameter & Group A & Group B & p value \\
Spasmodic sites & $\mathrm{N}=168$ & $26(35.6)$ & 0.872 \\
Radial artery & $58(34.5)$ & $36(49.3)$ & 0.436 \\
Brachial artery & $92(54.8)$ & $11(15.1)$ & 0.340 \\
Radial and brachial artery & $18(10.7)$ & $14(19.2)$ & $<0.0001$ \\
Hematoma in forearm & $7(4.2)$ & & $<0.0001$ \\
Guiding catheter crossover (yes or no) & & $28(38.4)$ & $<0.0001$ \\
Yes & $168(100.0)$ & $45(61.6)$ & $<0.0001$ \\
No & $0(0)$ & $\mathrm{N}=28$ & \\
Time consumption for Guiding & $\mathrm{N}=168$ & $4(14.3)$ & \\
catheter crossover & $157(93.5)$ & $19(67.9)$ & \\
55 min & $11(6.5)$ & $5(17.8)$ & \\
$>15$ min & $0(0)$ & & \\
\hline
\end{tabular}


Under the circumstances, it was hard for a $6 \mathrm{~F}$ guiding catheter to pass through the spasmodic segment, nevertheless, it was not necessary to change another 2.0 $\mathrm{mm}$-in-diameter balloon. On the basis of our experience, one $6 \mathrm{~F}$ guiding catheter and one $2.5 \mathrm{~mm}$-in-diameter balloon can also have a good match. Twelve cases in this investigation were the case.

\section{Discussion}

Trans-radial artery PCI has many advantages, so it has become the preferred approach for the interventional therapeutics of the majority of coronary heart diseases nowadays. However, the radial artery itself has some different characteristics, namely, its diameter is smaller than that of the femoral artery and prone to spasm; although the size of brachial artery is larger than that of the radial artery, it also has the feature of being easily spasmodic [3], so it has become a common problem in trans-radial artery PCI.

The vessel wall of radial artery mainly consists of the a1-adrenergic receptor and a small amount of $\beta$ receptor, which is extremely sensitive to the catecholamine in the circulation [4] [5]. Vessel puncture, sheath intubation, and catheterization can directly stimulate the vessels, thus exciting the sympathetic nerve system, increasing the catecholamine level in the blood, and causing the radial artery spasm. Patients in PCI will be nervous and anxious, and feel pain, so it will increase the catecholamine level in the circulation, which may also cause the radial artery spasm. The direct stimuli of operation itself, as well as the intraoperative and postoperative activation via neural fluid mechanism (including the increase of endothelin or catecholamine, etc.) are also related to the radial artery spasm [6]. The reasons of the brachial artery spasm are similar to those of the radial artery. In addition, according to the data of this study, it seems that the brachial artery is more prone to spasm, which may be related to its more sensitivity to the catecholamine and endothelin, but the affirmative reason is still subjected to further investigation.

The incidence of radial artery spasm in trans-radial artery PCI has significant differences in various studies. It was reported the incidence varied from 4.4\% [1] to $10 \%$ [7]. Some other studies had reported that if no preoperative vasodilator drugs were administrated to prevent artery spasm, the incidence even reached 20.4\% [8] [9]. Because the accurate analysis of the incidence of radial artery spasm was not our focus in this study, no exact incidence was statistically analysed. Reports on brachial artery spasm are less, and according to the authors' experience, its incidence is at least not lower than that of the radial artery spasm. In this study, the incidence of brachial artery spasm was higher than that of radial artery. Certainly, it still needs to further observe the two spasm models in clinical practice in the future. It should be noted that the incidence of the radial or (and) brachial artery spasm found in PCI may be lower than that in real-world interventional practice. Because a $5 \mathrm{~F}$ catheter is generally used in coronary angiography, and even if the radial and (or) brachial artery spasm occurs, 
the $5 \mathrm{~F}$ catheter can also pass through the spasmodic segment without being aware of its existence in most cases, at which time no angiography is generally performed for the confirmation, so that the incidence of the radial or (and) brachial artery spasm during PCI will be underestimated.

Once it is suspected that radial or brachial artery spasm occurs, angiography must be performed for confirmation. Actually, some suspected spasms are not the case. Under certain circumstances, the guiding wire and catheter do not follow the main stem of the radial or brachial artery, which may be inserted into a small artery of the elbow artery network (mostly into the radial recurrent artery or radial collateral artery). Because such vessels are smaller in diameter, a $5 \mathrm{~F}$ catheter sometimes can pass through, and almost all $6 \mathrm{~F}$ catheters cannot pass through the spasmodic or (and) dissected segment although most 0.035 "-indiameter guiding wire can easily cross over those relatively small arteries. Once the catheter is pushed into small artery following the guiding wire, resistance can be perceived, then angiography should performed immediately for confirmation. If it is confirmed that catheter is in the radial recurrent artery or radial collateral artery or other small arteries, the guiding wire and catheter should be re-adjusted so as to make them in the main vessel.

When radial or (and) brachial artery spasm occurs in PCI, the most critical step for guiding catheter crossing over the spasmodic segment is to maneuver the PCI wire carefully to pass through the spasmodic segment. Often in clinical practice, when the operator feels resistance while pushing guiding catheter and is aware of the existence of spasm, vessel dissection (sometimes serious dissection) probably has occurred. At this moment, operator should stop the operation immediately and withdraw the catheter a little and inject contrast medium through the guiding catheter. Often after angiography, contrast medium extravasation can be found, sometimes even the real vessel lumen cannot be identified.

Generally, there is no need to repeatedly administrate anti-spasmodic drugs at this time, and the guiding wire should be tried to pass through the spasmodic or (and) dissected segment as immediately as possible. It is important to select one soft-tip PCI guiding wire (the wire most commonly used is BMW or Runthrough guiding wire) for safe crossover of the spasmodic, especially the dissected segment; the guiding wire must be advanced gently during operation, and no force should be applied when encountering resistance. Only when the guiding wire passes through the spasmodic or dissected segment almost without any resistance can it be identified as being in the true lumen. Generally, it is easier for the guiding wire to pass through the spasmodic segment without dissection; however, if spasm is accompanied by serious dissection, there may be certain difficulties for the guiding wire to pass through, for the guiding wire may easily enter the false lumen. At this point, patience is important, and the true lumen must be carefully identified after injection of the diluted contrast medium; after that, the guiding wire should be advanced gently until it meets no significant resistance and passes through the spasmodic segment smoothly, which should be 
further confirmed by angiography. It may further aggravate dissection if the guiding wire was manipulated with violence, or even completely occlude the blood vessels. If the occlusion occurs in the brachial artery, it may cause acute upper limb ischemia. No such case occurred in this study, and the time consumption of spasmodic or (and) dissected segment crossover was within $5 \mathrm{mi}-$ nutes in most cases.

After the guiding wire successfully passes through the spasmodic or (and) dissected segment, the predilated balloon can then be advanced through the guiding wire. Generally, a 6F guiding catheter corresponds to a $2.0 \mathrm{~mm}$-in-diameter balloon, and a 7F guiding catheter corresponds to a $2.5 \mathrm{~mm}$-in-diameter balloon. Sometimes, a $6 \mathrm{~F}$ guiding catheter can also match a $2.5 \mathrm{~mm}$-in-diameter balloon, the inflation pressure of balloon should be kept at $6-8 \mathrm{~atm}$; thus the inflation pressure for a $6 \mathrm{~F}$ catheter and 2.0-mm balloon should be kept at $8-10$ atm. After the balloon is dilated, half of it should be kept in the guiding catheter while the rest half should be kept in the blood vessel. Because the diameter of the balloon tip is less than its body, the extracorporeal pushing of the guiding catheter may result in the so-called "plow"-like movement of the balloon tip, and the body part will have the expansion effect toward the spasmodic segment of the vessel. After dilation, the balloon body and the guiding catheter will be in closely contact as a whole, therefore the guiding catheter can easily pass through the radial or(and) brachial artery spasm or(and) dissection by the aid of the balloon. Because the balloon surface is very smooth, it will cause no obvious damage to the vascular intima. It has been proved in clinical practice that the guiding catheter can pass through the spasmodic or (and) dissected segment almost without any resistance in most cases.

Once radial or (and) brachial artery spasm occurs, the possibility of forearm hematoma will increase significantly, which is related to guiding catheter's damage to the spasmodic segment when it is pushed forcefully. Therefore, when completing the procedure, a 0.035 " guiding wire is usually used to guide the catheter's withdrawal from body. The catheter should be placed in the distal end of the previously spasmodic or dissected site, and repeated angiography should be performed so as to identify whether the spasm is relieved and whether contrast medium exudation occurs. It was demonstrated that most cases in this study exhibited different degrees of spasm relief, or even complete remission; furthermore, all the dissected segments had been closed, and no contrast medium extravasation occurred after PCI. The reason for spasm relief is considered as the result of the continuous outward force of the guiding catheter on the spasm segment. Similarly, the dissection closure is also related to the continuous outward force of the catheter on it. Patients who were routinely administrated anti-spasmodic drugs to relieve spasm had a certain proportion of forearm hematoma, which was the fact that the guiding catheter could not pass through serious dissection site and could not form a continuous outward force on dissection, it could not self-heal and resulted in blood extravasation thereafter. 
The application of PCI guiding wire and balloon assistance will not increase patients' financial burden because it is also the operational instrument necessary in PCI, so no additional instrument is required. On the contrary, because no other blood vessel needs to be punctured, it also needs not another sheath, and more importantly, it will reduce patients' suffering.

The combination with nitroglycerine and sodium nitroprusside has better effect than nitroglycerin alone in relieving the radial or (and) brachial artery [1]; if the combined effect is still not obvious, diltiazem can also be combined. However, it is obvious that the administration of drugs to relieve spasm often takes longer time, and there are still a large number of patients that spasm cannot be relieved effectively.

Since the beginning of 2014, small-scale clinical observation concerning this approach had been carried out and analysed by the first author, revealing the success rate as $100 \%$. Since then, this approach has been popularized and adopted by a number of interventionists in many domestic hospitals, and the success rates by different operators can also reach nearly $100 \%$.

It was an observational rather than a randomized controlled trial, the sample size was still not too large. Because the investigation was mainly focused on the immediate effect and safety of a relatively new approach, the long-term effect on clinical treatment outcomes was not evaluated. On the other hand, some other important index, for instance, the hospitalization cost, etc. were not compared because of much difference among hospitals across the country in hospital charge system, case selection bias between the two groups (patients enrolled in group B had a greater proportion of earlier hospitalization time). Further investigation in a larger patient population and in more operators is needed to corroborate the conclusions.

In short, the assistance of PCI wire and balloon is an extremely effective and safe approach for guiding catheter crossing over spasmodic or (and) dissected radial or(and) brachial artery in patients who PCI was undergone via radial artery access, which is more effective and safer than that of conventionally administrating anti-spasmodic drugs. So the approach of PCI wire and balloon assistance is worth being strongly recommended.

\section{Conflicts of Interest}

The authors have no conflicts of interest to declare.

\section{References}

[1] Gwon, H.C., Doh, J.H., Choi, J.H., Lee, S.H., Hong, K.P. and Park, J.E. (2006) A 5Fr Catheter Approach Reduces Patient Discomfort during Trans-Radial Coronary Intervention Compared with a 6Fr Approach: A Prospective Randomized Study. Journal of Interventional Cardiology, 19, 141-147. https://doi.org/10.1111/j.1540-8183.2006.00121.x

[2] Phillipe, F., Larrazet, F., Meziane, T. and Dibie A. (2004) Comparison of Trans-Radial vs Transfemoral Approach in the Treatment of Acute Myocardial Infarction with 
Primary Angioplasty and Abciximab. Catheterization Cardiovascular Interventions, 61, 67-73. https://doi.org/10.1002/ccd.10675

[3] Lin, Y.J., Liu, Y.B., Chu, C.C. and Tsai, C.W. (2006) Predictors of Brachial Artery Spasm during Trans-Radial Cardiac Catheterization. Acta Cardiologica Sinica, 22, 134-141.

[4] Kiemeneij, F., Laarman, G.J. and de Melker, E. (1995) Trans-Radial Artery Coronary Angioplasty. American Heart Journal, 129, 1-7. https://doi.org/10.1016/0002-8703(95)90034-9

[5] Wu, C.J., Lo, P.H., Chang, K.C., Fu, M., Lau, K.W. and Hung, J.S. (1997) Trans-Radial Coronary Angiography and Angioplasty in Chinese Patients. Catheterization Cardiovascular Interventions, 40, 159-163. https://doi.org/10.1002/(SICI)1097-0304(199702)40:2<159::AID-CCD8>3.0.CO;2-A

[6] Stella, P.R., Kiemeneij, F., Laarman, G.J., Odekerken, D., Slagboom, T. and van der Wieken, R. (1997) Incidence and Outcome of Radial Artery Occlusion Following Trans-Radial artery Coronary Angioplasty. Catheterization Cardiovascular Interventions, 40, 156-158.

https://doi.org/10.1002/(SICI)1097-0304(199702)40:2<156::AID-CCD7>3.0.CO;2-A

[7] Dai, J., Yao, M., Qiao, S.B., Yang, Y.J., Qin, X.W., An, X.G., Hou, Y. and Liu, H.B. (2004) An Analyzing the Causes of Procedural Failure and Complications of Coronary Angiography through Right Radial Approach. Chin Circ, 19, 175-177.

[8] Chen, C.W., Lin, C.L., Lin, T.K. and Lin, C.D. (2006) A Simple and Effective Regimen for Prevention of Radial Artery Spasm during Coronary Catheterization. Cardiology, 105, 43-47. https://doi.org/10.1159/000089246

[9] Coppola, J., Patel, T., Kwan, T., Sanghvi, K., Srivastava, S., Shah, S. and Staniloae, C. (2006) Nitroglycerin, Nitroprusside, or Both, in Preventing Radial Artery Spasm during Trans-Radial Artery Catheterization. Journal of Invasive Cardiology, 18, 155-158. 